\title{
Correction to: Passivity of Complex Dynamical Networks
}

\section{Correction to: \\ J.-L. Wang et al., Passivity of Complex Dynamical Networks, https://doi.org/10.1007/978-981-33-4287-3}

In the original version of the book, the following belated corrections have been incorporated:

(a) Reference 18 has been changed from "Neurocomputing, 7(9), 1782-1788" to "Neurocomputing, 72(7-9), 1782-1788" of Chap. 1.

(b) Corrections have been incorporated in Sects. 4.3.2 and 4.4.2 of Chap. 4.

(c) Corrections have been incorporated in Sect. 5.3.2 of Chap. 5.

(d) Corrections have been incorporated in Sect. 6.4.2 of Chap. 6.

(e) Corrections have been incorporated in Eqs. 7.4, 7.5, 7.7, 7.9, 7.16, 7.17 and 7.19 of Chap. 7.

(f) In Chap. 8, the sentence "As a natural extension of the existing models, we introduce a new CDN model with time delay which describes a cooperative interaction of $\mathrm{N}$-species that benefits each other in a bounded habitat $\Theta$." has been changed to "As a natural extension of the existing models, we introduce a new $\mathrm{CDN}$ model with time-delay which describes a cooperative interaction of $\mathrm{N}$-species that benefits each other in a bounded habitat $\Theta$."

(g) In Chap. 9, (a) the sentence "In [27], the authors presented a switched coupled uncertain NN, and derived several delay-independent and delay-dependent

\footnotetext{
The updated version of these chapters can be found at https://doi.org/10.1007/978-981-33-4287-3_1 https://doi.org/10.1007/978-981-33-4287-3_4 https://doi.org/10.1007/978-981-33-4287-3_5 https://doi.org/10.1007/978-981-33-4287-3_6 https://doi.org/10.1007/978-981-33-4287-3_7 https://doi.org/10.1007/978-981-33-4287-3_8 https://doi.org/10.1007/978-981-33-4287-3_9 https://doi.org/10.1007/978-981-33-4287-3
} 
passivity criteria for such a network model." has been changed to "In [27], the authors presented a switched coupled uncertain "NNs", and derived several delay-independent and delay-dependent passivity criteria for such a network model."; (b) the placement of Equation (9.12) has been corrected; (c) the sentence "Figure 9.1 presents the evolution processes of the norms of error vectors $9 \mathrm{i}(\mathrm{x}, \mathrm{t})$, output vectors yi $(x, t)$ and input vectors ui $(x, t), i=1,2,3,4,5$, 6. Figure 9.2 illustrates the evolution tendencies of adaptive feedback gains." has been changed to "Fig. 9.1 presents the evolution processes of the norms of error vectors $9 \mathrm{i}(\mathrm{x}, \mathrm{t})$, output vectors yi $(\mathrm{x}, \mathrm{t})$ and input vectors ui $(\mathrm{x}, \mathrm{t}), \mathrm{i}=1,2,3,4,5$, 6. Fig. 9.2 illustrates the evolution tendencies of adaptive feedback gains."; and (d) Chap. 9, Page 239, the sentence "Figure 9.3 shows the evolution processes of the norms of error vectors $9 \mathrm{i}(\mathrm{x}, \mathrm{t}), \mathrm{i}=1,2,3,4,5,6$. Evidently, the error vectors $9 \mathrm{i}(\mathrm{x}, \mathrm{t}), \mathrm{i}=$ 1, 2, 3, 4, 5, 6 can converge to zero. Figure 9.4 displays the evolution tendencies of adaptive feedback gains." has been changed to "Fig. 9.3 shows the evolution processes of the norms of error vectors $9 \mathrm{i}(\mathrm{x}, \mathrm{t}), \mathrm{i}=1,2,3,4,5,6$. Evidently, the error vectors $9 \mathrm{i}(\mathrm{x}, \mathrm{t}), \mathrm{i}=1,2,3,4,5,6$ can converge to zero. Fig. 9.4 displays the evolution tendencies of adaptive feedback gains."

The book and the chapters have been updated with the changes. 